\title{
The levels of urinary joining chain containing IgG immune complexes are associated with disease severity in IgA nephropathy
}

\section{Guanhong Li}

Division of Nephrology, Department of Internal Medicine, Peking Union Medical College Hospital, Chinese Academy of Medical Sciences \& Peking Union Medical College

\section{Cai Yue}

Division of Nephrology, Department of Internal Medicine, Peking Union Medical College Hospital, Chinese Academy of Medical Sciences \& Peking Union Medical College

\section{Xiaohong Fan}

Division of Nephrology, Department of Internal Medicine, Peking Union Medical College Hospital, Chinese Academy of Medical Sciences \& Peking Union Medical College

\section{Yubing Wen}

Division of Nephrology, Department of Internal Medicine, Peking Union Medical College Hospital, Chinese Academy of Medical Sciences \& Peking Union Medical College

\section{Gang Chen}

Division of Nephrology, Department of Internal Medicine, Peking Union Medical College Hospital, Chinese Academy of Medical Sciences \& Peking Union Medical College

\section{Yan Qin}

Division of Nephrology, Department of Internal Medicine, Peking Union Medical College Hospital, Chinese Academy of Medical Sciences \& Peking Union Medical College

\section{Ruitong Gao ( $\square$ gaoruitong@gmail.com )}

Devison of Nephrology, Department of Internal Medicine, Peking Union Medical College Hospital, Chinese Academy of Medical Sciences \& Peking Union Medical College, Beijing, China https://orcid.org/0000-0002-2117-5032

\section{Xuemei Li}

Division of Nephrology, Department of Internal Medicine, Peking Union Medical College Hospital, Chinese Academy of Medical Sciences \& Peking Union Medical College

\section{Research article}

Keywords: Joining chain, IgA nephropathy, immune complexes to creatinine ratio, urine

Posted Date: November 4th, 2019 
DOI: https://doi.org/10.21203/rs.2.13663/v2

License: (c) (1) This work is licensed under a Creative Commons Attribution 4.0 International License. Read Full License 


\section{Abstract}

Background: Circulating levels of aberrantly glycosylated $\operatorname{lgA} 1$ and its immune complexes (IgA/IgG-IC) are elevated and correlated with disease severity in $\lg A$ nephropathy (IgAN). The pathologic IgA containing immune complexes deposits in the kidney were found to be in dimeric or polymeric forms, suggesting that those deposited IgA immune complexes contain joining chain, which is critical for the multimerization and transportation of $\lg \mathrm{A}$. Both the dimeric $\lg \mathrm{A}$ and polymeric $\lg \mathrm{A}$ can cause renal damage by inducing the release of cytokines from mesangial cells. We aimed to investigate the urinary $\mathrm{J}$ chain containing IgG immune complexes/creatinine ratio (UJGCR) and analyze its relationship with disease severity in IgAN.

Methods: The UJGCR were measured by sandwich enzyme linked immunosorbent assay in 26 patients with IgAN, 31 patients with other kidney diseases and 32 healthy volunteers.

Results: The levels of UJGCR were higher in patients with IgAN than that in non-IgAN patients $(P=0.006)$ and healthy volunteers $(P<0.0001)$. Importantly, receiver operating characteristics curve analysis confirmed that UJGCR had good discrimination between non-IgAN patients and IgAN patients. The levels of UJGCR positively correlated with 24-hour urinary protein excretion $(r=0.63, P=0.0006)$, serum creatinine $(r=0.55, P=0.003)$, and negatively correlated with estimated glomerular filtration rate $(r=-0.61$, $\mathrm{P}=0.0008)$ in IgAN. Furthermore, the levels of UJGCR were higher in IgAN patients with IgA mesangial deposition of $(+/++)$ than patients with $(+++/++++)$. And IgAN patients with tubular atrophy/interstitial fibrosis showed higher levels of UJGCR than that without $(P=0.03)$. Similarly, the levels of urinary IgA$\mathrm{lg}$ /creatinine ratio (UAGCR) were also found to be elevated and associated with clinical and pathological parameters as UJGCR in IgAN. Besides, significant correlation between the levels of UJGCR and UAGCR was shown, suggesting UJGCR was mainly composed of J-lgA-lgG.

Conclusions: The levels of UJGCR are associated with disease severity in IgAN. UJGCR is a potential biomarker for both glomerular and tubulointerstitial lesions in IgAN.

\section{Background}

$\lg$ A nephropathy $(\operatorname{IgAN})$ is one of the most common glomerulonephritis worldwide [1, 2]. IgAN is associated with a poor prognosis, over $40 \%$ of patients with IgAN progress slowly to end-stage renal failure (ESRD) 30 to 40 years after diagnosis [3]. Regular measurement of kidney injury and disease progression is crucial for patients with IgAN during the follow-up period. Although proteinuria, hypertension, renal dysfunction, and a few histological features have been identified as parameters for disease severity of IgAN [4-6], these biomarkers are not always specific for an individual. Thus, noninvasive and more specific biomarkers are still needed to accurately access the disease severity in IgAN.

IgAN is an immune complexes mediated glomerulonephritis characterized by the presence of IgA containing immune complexes deposits in the mesangial area [7]. Although the pathogenesis of IgAN is still under investigation, it has been suggested that galactose-deficient IgA1 (Gd-lgA1) and its 
corresponding immune complexes (IgA/lgG-IC) were found to be elevated and in IgAN [8-12]. Berthoux et al. reported that serum Gd-IgA1 and IgA/IgG-IC levels wereare associated with progression in IgAN [9]. And Suzuki et al. found that these serum biomarkers wereare associated with the degree of hematuria and proteinuria in IgAN [11]. Evidences from recent researches suggested that Gd-IgA1 and IgA/IgG-IC accumulated in the glomerular mesangium to instigate renal damage $[13,14]$.

Researches from the past decades led to an agreement that these $\lg A$ deposits in the kidney wereare in dimeric or polymeric forms [15-17], revealing that these deposited IgA immune complexes contained a small disulfide-linked polypeptide of $15 \mathrm{kDa}$ known as the joining chain ( $\mathrm{J}$ chain). It is generally agreed that $\mathrm{J}$ chain regulates the multimerization of $\lg \mathrm{A}$ and $\lg \mathrm{M}$ comparing to $\lg \mathrm{G}$, forming secretory $\lg \mathrm{A}(\mathrm{s} \lg \mathrm{A}$ ), polymeric $\lg A(p \lg A)$, and pentameric $\lg M(p \lg M)[18-20]$. $J$ chain is also required for the transportation of slgA across the mucosal epithelium, preventing attachment of bacteria and viruses to mucous membranes [20]. It is well known that the classic manifestation of IgAN is episodic hematuria with or without proteinuria following mucosal infection. It is possible that mucosal immunity dysregulation in IgAN might lead to the increased synthesis of slgA which is transported across the mucosa with the help of $\mathrm{J}$ chain and thus reaches the circulation and deposit in mesangium. Several investigations have revealed that the serum levels of slgA and plgA wereare elevated in IgAN [21-24]. And J chain had been found in renal biopsy specimens of IgAN patients using anti-J chain polyclonal antibody or antisera [15, $16,25]$. Bothe the dDimeric IgA and plgA both couldcan cause renal damage by inducing the release of cytokines, including interleukin-6 (IL-6), tumor necrosis factor- a (TNF- $a)$, and transforming growth factor- $\beta$ (TGF- $\beta$ ) by mesangial cells [26-29]. It is possible that a fraction of circulating $\mathrm{J}$ chain containing immune complexes deposits in the kidneys of IgAN patients are excreted into the urine and thus represent as non-invasive disease-specific biomarkers in IgAN. However, only one previous study found that the levels of urinary IgA-lgG complexes were significantly higher in IgAN patients [30]. The value of urinary IgA-lgG immune complexes for accessing disease severity in IgAN remains unclear. In addition, urinary $\mathrm{J}$ chain in IgAN has not been reported and it is not known whether the levels of urinary $\mathrm{J}$ chain containing immune complexes are associated with clinical or pathological severity in IgAN.

In this study, we detected urinary $\mathrm{J}$ chain containing $\lg \mathrm{G}(\mathrm{J}-\mathrm{lgG})$ immune complexes by sandwich enzyme linked immunosorbent assay (ELISA) with anti-J chain monoclonal antibody (mAb). We analyzed the association between the levels of urinary J-IgG immune complexes and clinicopathological parameters of IgAN at the time of renal biopsy.

\section{Methods}

\section{Patients}

We enrolled 26 patients with biopsy-proven primary IgAN (IgAN group), 31 patients with other renal diseases (Disease control group, DC group) and 32 healthy volunteers (Healthy control group, HC group). All patients admitted to Peking Union Medical College Hospital between April 2016 and September 2016. The histologic diagnosis of IgAN was based upon the demonstration of mesangioproliferative changes 
on light microscopy and the concomitant presence of predominant or codominant mesangial deposition of IgA. Patients with lupus nephritis $(L N)$, Henoch-Schönlein purpura, liver cirrhosis and other secondary causes of IgAN were excluded from the study. The diagnoses of DC group included membranous

nephropathy $(n=15)$, minimal change nephropathy $(n=3), L N(n=1)$, diabetic nephropathy $(n=2)$, focal segmental glomerulosclerosis $(n=2)$, chronic pyelonephritis or interstitial nephritis $(n=4)$, hypertensive renal damage $(n=1)$ and sclerosing glomerulonephritis $(n=2)$, obesity-related glomerulopathy $(n=1)$.

\section{Clinical and pathological manifestations}

The demographic and clinical parameters of patients, including age, gender, blood pressure (BP), urinary red blood cells count, serum creatinine ( $\mathrm{SCr}$ ), urine creatinine ( $\mathrm{UCr}$ ) and 24-hour urinary protein excretion (24hUPro) were obtained immediately before renal biopsy. Estimated glomerular filtration rate (eGFR) was calculated using the Chronic Kidney Disease Epidemiology Collaboration (CKD-EPI) equation [31].

Medication history, including the usage of cortisone, immunosuppressant and renin-angiotensin system (RAS) blockers such as angiotensin-converting enzymes inhibitors (ACE-Is) and angiotensin II receptor blockers (ARBs) was also recorded.

\section{Renal biopsies}

For each patient, the diagnosis of IgAN was based on histologic assessment of renal biopsy tissue with hematoxylin and eosin, periodic acid-Schiff and Masson's trichrome for light microscopy and staining with antibodies against $\lg \mathrm{A}, \lg \mathrm{G}, \lg \mathrm{M}, \mathrm{C} 1 \mathrm{q}, \mathrm{C} 3, \mathrm{C} 4$ and fibrinogen for immunofluorescence. IgAN was defined by the presence of at least $1+$ (range, $0-4) \lg A$ mesangial deposits as dominant or codominant immunoglobulins on immunofluorescence microscopy performed on frozen tissue. Pathological features were evaluated according to updated Oxford classification [32] and Lee grading [33], which were graded by a pathologist blinded to patients' clinical data.

\section{Urine samples}

Spot morning urine samples from patients were collected on the same day of renal biopsy. All samples were centrifuged at $3000 \mathrm{rmp} / \mathrm{min}$ for $10 \mathrm{~min}$ to remove cellular components and the supernatant was kept at $-80^{\circ} \mathrm{C}$ until use. Spot morning urine from healthy controls were also collected and prepared in the same manner as for patients.

Generation of anti-J chain monoclonal antibody

J chain-GST recombinant peptide IGJ-pGEX-4T-1 (NCBI ID for J chain sequence: NM_144646) was synthesized and purified as an antigen to immunize BALB/c mice. Candidate hybridomas were established from splenocytes (Absea Biotechnology Ltd., Beijing, China). Hybridomas that produce anti-J 
chain monoclonal antibodies were selected by ELISA with the recombinant antigen peptide, by Dot-blot with the plgA purified from multiple myeloma patients and saliva that contains $\mathrm{J}$ chain-sigA by Western Blot.

\section{Detection of urinary J-IgG and IgA-IgG immune complexes by ELISA}

To measured urinary immune complexes, ninety-six well microtiter plates were coated with anti-J chain mAb or anti-IgA mAb (Absea Biotechnology Ltd., Beijing, China) in carbonate-bicarbonate buffer, pH 9.6, overnight at $4{ }^{\circ} \mathrm{C}$. The plates were washed three times with phosphate buffered saline (PBS) containing $0.05 \%$ Tween 20 (PBST) and then blocked with $200 \mu \mathrm{L} /$ well of $2 \%$ bovine serum albumin (BSA) for $2 \mathrm{~h}$ at $37^{\circ} \mathrm{C}$. The urine samples were added to the same plate and incubated for $3 \mathrm{~h}$ at $37^{\circ} \mathrm{C}$. Then the plates were washed and HRP-conjugated mouse anti-human IgG (Absea Biotechnology Ltd., Beijing, China) was added and incubated for $1 \mathrm{~h}$ at $37^{\circ} \mathrm{C}$. After washing, the color was developed using TBS (Amresco, Solon, USA) as a substrate, and the absorbance was measured at $450 \mathrm{~nm}$ with a microplate reader (BioTek Synergy $4 \llbracket$ Winooski, VT, USA).

Levels of urinary J-IgG (UJGCR) and IgA-IgG (UAGCR) immune complexes were standardized to the UCr concentration to adjust for differences of urine flow rate.

\section{Statistical analysis}

Statistical calculations were performed using SPSS for Windows, version 20.0 (SPSS, Chicago, IL) and Graph Pad Prism 5.0 (Graph Pad Software Inc., San Diego, CA). Data are presented as mean values \pm SD, medians (interquartile range) or frequency in percent according to the types of variables. The MannWhitney U-test was used for statistical comparisons between two groups. Receiver operator characteristic curve (ROC) analysis was performed to assess the value of urine markers in differentiating between patients with IgAN and combined controls. Correlation analyses were performed using Spearman's correlation analyses. All tests were two-sided and a P-value $<0.05$ was considered statistically significant.

\section{Results}

\section{Demographic and clinical characteristics of the participants}

Demographic and clinical characteristics of the study subjects wereare summarized in Table 1. Overall, our study population consisted of 26 patients with IgAN ( 9 males and 17 females), 31 patients with other renal diseases ( 16 males and 15 females), and 32 healthy volunteers ( 22 males and 10 females). The mean age was $41(32-47)$ years, $46(29-60)$ years, and 46(29-60) years for the groups of IgAN, DC and $\mathrm{HC}$, respectively. Compared to patients with other renal diseases, the values of $24 \mathrm{hUPro}$ were significantly 
lower [1.32 (0.65-3.48) g/24h versus $4.05(1.46-5.97) \mathrm{g} / 24 \mathrm{~h}, P=0.01]$ and serum albumin were higher $[39.00(33.00-43.00) \mathrm{g} / \mathrm{L}$ versus $30.00(25.00-40.00)]$ in patients with IgAN.

A total of $16(61.5 \%)$ patients with IgAN were treated with RAS blockers, $8(30.8 \%)$ were treated with immunosuppressive therapy, and 4(15.4\%) received corticosteroids. In DC group, 17(54.8\%) were treated with RAS blockers, 15(48.4\%) were treated with immunosuppressive therapy, and 18(58.1\%) received corticosteroids. The utilization rate of corticosteroids in patients with IgAN was significant lower than that in patients of patients with other kidney diseases. However, the distribution of gender, age, and treatments (immunosuppressants and ACE-Is / ARBs) did not differ between IgAN group and DC group.

\section{UJGCR were elevated in patients with IgAN compared with controls}

The levels of UJGCR were significantly higher in patients with IgAN than that in patients with other renal diseases $(0.31 \pm 0.200 D 450 \mathrm{~nm} / \mathrm{mmol} / \mathrm{L}$ Cr versus $0.19 \pm 0.170 D 450 \mathrm{~nm} / \mathrm{mmol} / \mathrm{L} \mathrm{Cr}, P=0.006)$ and healthy subjects $(0.31 \pm 0.200 D 450 \mathrm{~nm} / \mathrm{mmol} / \mathrm{L}$ Cr versus $0.04 \pm 0.040 D 450 \mathrm{~nm} / \mathrm{mmol} / \mathrm{L} \mathrm{Cr}, P<0.0001)$. And the levels of UAGCR were also significantly higher in patients with IgAN than that in patients with other kidney diseases $(P=0.004)$ and healthy subjects $(P<0.0001)$ (Fig. 1$)$. In addition, we found that the values of these urinary markers did not differ between IgAN patients with corticosteroid therapy $(n=4)$ and those without $(n=22)$ (data not shown). We also found that the values of urinary markers did not differ between IgAN patients with immunosuppressant therapy $(n=7)$ and those without $(n=19)$ (data not shown).

\section{ROC comparing UJGCR in distinguishing patients with IgAN from patients with other renal diseases}

ROC analysis confirmed good discrimination between patients with IgAN and patients with other renal diseases for elevated levels of UJGCR (AUC, $0.71 ; 95 \% \mathrm{Cl}, 0.58-0.85 ; P=0.006)$ and UAGCR (AUC, 0.72; $95 \% \mathrm{Cl}, 0.59-0.85 ; P=0.04$ ) (Fig.2). The respective optimal derived cut-off values were 0.1491 OD450nm/mmol/L Cr for UJGCR (Sensitivity: $84.6 \%$; Specificity: $58.1 \%$ ) and 0.0877 OD450nm/mmol/L Cr for UAGCR (Sensitivity: $84.6 \%$; Specificity: $58.1 \%$ ).

\section{Association between clinical parameters and urinary immune complex in patients with IgAN}

In patients with IgAN, the levels of UJGCR were correlated positively with the levels of 24hUPro and SCr, as well asand negatively correlated with eGFR $(r=0.63, P=0.0006 ; r=0.55, P=0.003 ; r=-0.61, P=$ 0.0008 , respectively) (Fig.3a-C). Likewise, the significant correlation between the levels of UAGCR and 
24hUPro, SCr, and eGFR ( $r=0.58, P=0.002 ; r=0.67, P=0.0002 ; r=-0.75, P<0.0001$, respectively).

(Fig.3d-f) were also observed. There was no significant correlation ofbetween U-RBC with UJGCR andor UAGCR and U-RBC (data not shown).

A significant correlation between UAGCR and UJGCR $(r=0.80, P<0.0001)$ was observed in patients with IgAN patients (see Additional file 1).

\section{Association between pathological parameters and UJGCR in patients with IgAN}

We examined the association between urinary immune complexes and different histological lesions. The intensity of the immunofluorescence of renal IgA deposition was graded as none $(-)$, trace $( \pm),(+),(++)$, $(+++)$ and (++++). Surprisingly, the levels of UJGCR [IgA deposition of $(+/++): 0.36(0.22-0.49)$ $\mathrm{nm} / \mathrm{mmol} / \mathrm{L}$ vs. IgA deposition of (+++/+++++): $0.16(0.08-0.22)$ OD $450 \mathrm{~nm} / \mathrm{mmol} / \mathrm{L} \mathrm{Cr}, P=0.0007]$ were higher in IgAN patients with IgA mesangial deposition of $(+/++)$ than patients with $(+++/++++)$ (see Additional file 1).

Regarding Lee grading, UAGCR and UJGCR were significantly higher in patients of grade IV $(n=18)$ than that in grade II and III ( $n=8$ in total) (see Additional file 3). We also evaluated the tubular atrophy/interstitial fibrosis grade according to Oxford classification. The levels of UJGCR [T0: 0.16 (0.10$0.31) \mathrm{OD} 450 \mathrm{~nm} / \mathrm{mg} \mathrm{Cr}$ vs. T1/T2: $0.36(0.21-0.48) \mathrm{OD} 450 \mathrm{~nm} / \mathrm{mmol} / \mathrm{L} \mathrm{Cr}, P=0.03$ ] were significantly higher in patients with tubular atrophy/interstitial fibrosis $(n=11)$ than that without $(n=15$ in total). Similarly, the levels of UAGCR were also significantly elevated as UJGCR between two subgroups (see Additional file 3).

However, UJGCR and UAGCR of IgAN patients with different grades of mesangial hypercellularity, segmental glomerulosclerosis and cellular/fibrocellular crescents did not show any significant differences (data not shown).

In patients with other renal diseases, the UJGCR levels were positively correlated with the levels of $\mathrm{SCr}$, but no correlation with 24hUPro or eGFR. And UAGCR levels were correlated with SCr and eGFR, but no correlation of UAGCR levels with 24hUPro was shown (see Additional file 4).

\section{Discussion}

In the present study, our data showed that the levels of UJGCR and UAGCR were markedly elevated in patients with IgAN compared with patients with other kidney diseases and healthy subjects. These elevated urinary immune complexes couldan distinguish patients with IgAN from patients with other renal diseases, as shown by the AUC in the ROC analysis. Furthermore, we demonstrated for the first time that the elevated UJGCR and UAGCR levels correlated with clinical and pathological injury in patients with IgAN. 
IgAN is characterized by the presence of IgA containing immune complexes deposits in the mesangium [7]. And studies have confirmed that Gd-IgA1 containing IgG complexes exhibited stronger stimulation of mesangial cell proliferation comparing to uncomplexed Gd-IgA1 [34-36]. Gd-IgA1 and IgA/IgG-IC in the circulation were found to be elevated and associated with progression and disease severity in IgAN [812]. These evidences strongly suggest that IgAN is a kind of immune complexes mediated glomerulonephritis. It is generally accepted that the $\lg A$ deposits in the kidney are in dimeric or polymeric forms [15-17, 23, 37], suggesting that those deposited IgA immune complexes contain $J$ chain. Previous studies had demonstrated that $\mathrm{J}$ chain wasis required for the multimerization of $\lg \mathrm{A}$ forming $\operatorname{sg} \mathrm{A}$ and plgA [18-20]. And $\mathrm{J}$ chain can also mediates the transportation of slgA and plgA through binding to $\lg$ receptor (plgR) on the basolateral side of epithelial cells [38-40]. It is possible that excessive production of galactose deficient slgA after mucosal infection might be transported through the epithelium under the help of $\mathrm{J}$ chain, forming immune complexes with its glycan-specific $\lg \mathrm{G}$ or $\lg \mathrm{A}$ autoantibodies in the circulation, and thus deposit in the mesangium. A part of these immune complexes might pass through the filter barrier of injury glomeruli and thus enter the urine.

Karel et al have reported that the urinary concentrations of UAGCR were elevated and correlated with the magnitude of proteinuria [30], which is consistent with our study. But neither did they analyze the relationship between UAGCR and pathological lesions in IgAN, nor did they measure urinary $\mathrm{J}$ chain in IgAN. In this study, we found that the elevated levels of UJGCR and UAGCR were not only correlated with 24hUPro, but also correlated well with kidney function in IgAN, which are well-known factors in predicting disease severity and renal outcome for the development of ESRD in IgAN patients.

To the best of our knowledge, this is the first study to demonstrate that UJGCR and UAGCR correlate with pathological parameters in patients with IgAN. In this study, we analyzed the associations between urinary immune complexes and histopathological phenotypes with disease predictive significance according to the Oxford classification, which indicated that the elevated levels of UJGCR and UAGCR in IgAN patients correlated with tubular atrophy/interstitial fibrosis. Moreover, surprisingly, we found that UJGCR and UAGCR were all higher in IgAN patients with less renal IgA deposition than patients with a more intense renal IgA deposition, which strongly suggests that these immune complexes deposits in the mesangium could cross the filtration barrier and thus be excreted into the urine. Taken together, it seems most likely that J-lgG and IgA-lgG immune complexes in the urine detected in the present study came from glomerular mesangium and injured the kidney, especially renal tubular epithelial cells.

More interestingly, this current study found significant correlations between UJGCR and UAGCR in IgAN. Based on our observation, we postulate UJGCR were mainly composed of J-lgA-lgG.

In clinical setting, renal biopsy is the only golden procedure to diagnose and evaluate disease activity in IgAN, but this being an invasive procedure which is impractical to repeat. In addition, 24hUPro, urine protein/creatinine excretion (UPCR), SCr and hematuria are often used to evaluate the severity of IgAN which could not always distinguish patients of IgAN from patients with other renal diseases. Thus, 
UJGCR measurement may be of particular value in diagnosing and evaluating the deterioration of renal injury in IgAN, avoiding more invasive procedures when not necessary.

There were some limitations in our research. First, urinary immune complexes were measured at a single time-point and it is unclear whether the levels of urinary immune complexes may fluctuate on different occasions. It is widely accepted that spot urinary protein/creatinine ratio predicts actual 24UPro with reasonable accuracy $[41,42]$. So the levels of UJGCR and UAGCR in our study were all standardized to the $\mathrm{UCr}$ to adjust for differences of urine flow rate. Secondly, further prospective studies in a larger population of patients are warranted to measure levels of serum $\mathrm{J}$ chain, urinary $\mathrm{J}$ chain, renal $\mathrm{J}$ chain, and $\mathrm{mRNA}$ expression of $\mathrm{J}$ chain to support our hypothesis.

\section{Conclusions}

In summary, our data demonstrate that UJGCR is potential specific biomarkers in IgAN. Higher levels of UJGCR not only related to relatively severe clinical features, but also pathologic involvement on renal biopsy, especially tubulointerstitial injury. These findings support the notion that plgA containing immune complexes might play an important role in the pathogenesis of IgAN. In conclusion, UJGCR could be a useful and reliable biomarker for evaluating the severity of renal injury in IgAN.

\section{Abbreviations}

ACE-Is: angiotensin-converting enzymes inhibitors; ARBs: angiotensin II receptor blockers; BP: blood pressure; BSA: bovine serum albumin; C: cellular/fibrocellular crescents; CKD-EPI: Chronic Kidney Disease Epidemiology Collaboration; DC group: disease control group; E: endocapillary hypercellularity; ESRD: end-stage renal failure; Gd-IgA1: galactose-deficient IgA1; HC group: healthy control group; IgAN: $\lg A$ nephropathy; IL-6: interleukin-6; J chain: joining chain; M: mesangial hypercellularity score; slgA: secretory IgA; mAb: monoclonal antibody (mAb); plgA: polymeric lgA; plgM: pentameric lgM; plgR: Ig receptor; ROC: Receiver operator characteristic curve; RAS: renin-angiotensin system; S: segmental glomerulosclerosis; SCr: serum creatinine; slgA: secretory IgA; T: tubular atrophy/interstitial fibrosis; TNF$\alpha$ : tumor necrosis factor- $\alpha$; TGF- $\beta$ : transforming growth factor- $\beta$; UJGCR: urinary $\mathrm{J}$ chain containing IgG immune complexes; UCr: urine creatinine; UAGCR: urinary IgA-IgG immune complexes; UPCR: urine protein/creatinine excretion 24hUPro: 24-hour urinary protein excretion

\section{Declarations}

\section{Ethics approval and consent to participate}

The study protocol was approved by the Peking Union Medical College Hospital Ethics Committee and the study was conducted in accordance with the World Medical Association Declaration of Helsinki.

\section{Consent for publication}


All participants provided written informed consent to participate in this study.

\section{Availability of data and materials}

The datasets used and/or analysed during the current study are available from the corresponding author on reasonable request.

\section{Competing interests}

The authors declare that they have no competing interests.

\section{Funding}

This study was supported by Beijing Municipal Natural Science Foundation (grant No.7192165). The funders had no role in the design of the study, data collection, data analysis, interpretation of data, and writing the manuscript.

\section{Authors contributions}

G. H. L. was involved in the study design, sample collection, biomarkers measurement\analysis and interpretation of the data, as well as drafting the manuscript. C. Y., X. H. F., Y. Q. and G. C. were involved in patient enrollment and clinical data collection. Y. B.W made the pathological confirmations of IgAN. R. T. G. and X. M.L are the corresponding authors who conceived the study and participated in the study design, interpretation of results. All authors critically revised the manuscript as well as reading and approving the final version prior to submission.

\section{Acknowledgements}

Not applicable.

\section{References}

1.Julian BA, Waldo FB, Rifai A, Mestecky J: IgA nephropathy, the most common glomerulonephritis worldwide. A neglected disease in the United States? The American journal of medicine 1988, 84(1):12932.

2.D'Amico G: The commonest glomerulonephritis in the world: IgA nephropathy. The Quarterly journal of medicine 1987, 64(245):709-27. 
3.Yeo SC, Cheung CK, Barratt J: New insights into the pathogenesis of IgA nephropathy. Pediatr Nephrol 2017. doi:10.1007/s00467-017-3699-z

4.Barratt J, Feehally J: IgA nephropathy. Journal of the American Society of Nephrology: JASN 2005, 16(7):2088-97. doi:10.1681/asn.2005020134

5.Coppo R, D'Amico G: Factors predicting progression of IgA nephropathies. Journal of nephrology 2005, 18(5):503-12.

6.Goto M, Wakai K, Kawamura T, Ando M, Endoh M, Tomino Y: A scoring system to predict renal outcome in IgA nephropathy: a nationwide 10-year prospective cohort study. Nephrology, dialysis, transplantation: official publication of the European Dialysis and Transplant Association - European Renal Association 2009, 24(10):3068-74. doi:10.1093/ndt/gfp273

7.Berger J, Hinglais N: [Intercapillary deposits of IgA-IgG]. Journal d'urologie et de nephrologie 1968, 74(9):694-5.

8.Suzuki H, Fan R, Zhang Z, Brown R, Hall S, Julian BA et al: Aberrantly glycosylated IgA1 in IgA nephropathy patients is recognized by IgG antibodies with restricted heterogeneity. The Journal of clinical investigation 2009, 119(6):1668-77. doi:10.1172/jci38468

9.Berthoux F, Suzuki H, Thibaudin L, Yanagawa H, Maillard N, Mariat C et al: Autoantibodies targeting galactose-deficient IgA1 associate with progression of IgA nephropathy. Journal of the American Society of Nephrology: JASN 2012, 23(9):1579-87. doi:10.1681/asn.2012010053

10.Yanagawa H, Suzuki H, Suzuki Y, Kiryluk K, Gharavi AG, Matsuoka K et al: A panel of serum biomarkers differentiates IgA nephropathy from other renal diseases. PloS one 2014, 9(5):e98081.

doi:10.1371/journal.pone.0098081

11.Suzuki Y, Matsuzaki K, Suzuki H, Okazaki K, Yanagawa H, leiri N et al: Serum levels of galactosedeficient immunoglobulin (Ig) A1 and related immune complex are associated with disease activity of IgA nephropathy. Clinical and experimental nephrology 2014, 18(5):770-7. doi:10.1007/s10157-013-09216

12.Berthelot $\mathrm{L}$, Robert $\mathrm{T}$, Vuiblet $\mathrm{V}$, Tabary $\mathrm{T}$, Braconnier $\mathrm{A}$, Drame $\mathrm{M}$ et al: Recurrent IgA nephropathy is predicted by altered glycosylated IgA, autoantibodies and soluble CD89 complexes. Kidney international 2015. doi:10.1038/ki.2015.158

13.Suzuki H, Kiryluk K, Novak J, Moldoveanu Z, Herr AB, Renfrow MB et al: The pathophysiology of IgA nephropathy. Journal of the American Society of Nephrology: JASN 2011, 22(10):1795-803. doi:10.1681/asn.2011050464

14.Giannakakis K, Feriozzi S, Perez M, Faraggiana T, Muda AO: Aberrantly glycosylated IgA1 in glomerular immune deposits of IgA nephropathy. Journal of the American Society of Nephrology: JASN 
15.Komatsu N, Nagura H, Watanabe K, Nomoto Y, Kobayashi K: Mesangial deposition of J chain-linked polymeric IgA in IgA nephropathy. Nephron 1983, 33(1):61-4.

16.Donini U, Casanova S, Zini N, Zucchelli P: The presence of $\mathrm{J}$ chain in mesangial immune deposits of IgA nephropathy. Proceedings of the European Dialysis and Transplant Association European Dialysis and Transplant Association 1983, 19:655-62.

17.0ortwijn BD, Rastaldi MP, Roos A, Mattinzoli D, Daha MR, van Kooten C: Demonstration of secretory IgA in kidneys of patients with IgA nephropathy. Nephrology, dialysis, transplantation: official publication of the European Dialysis and Transplant Association - European Renal Association 2007, 22(11):3191-5. doi:10.1093/ndt/gfm346

18.Brandtzaeg P: Presence of $\mathrm{J}$ chain in human immunocytes containing various immunoglobulin classes. Nature 1974, 252(5482):418-20.

19.Brandtzaeg P: Immunochemical studies on free and bound $\mathrm{J}$ chain of human IgA and IgM. Scandinavian journal of immunology 1975, 4(5-6):439-50.

20.Castro CD, Flajnik MF: Putting J chain back on the map: how might its expression define plasma cell development? Journal of immunology 2014, 193(7):3248-55. doi:10.4049/jimmunol.1400531

21.Lozano L, Garcia-Hoyo R, Egido J: IgA nephropathy: association of a history of macroscopic hematuria episodes with increased production of polymeric IgA. Nephron 1987, 45(2):98-103. doi:10.1159/000184087

22.Jones CL, Powell HR, Kincaid-Smith P, Roberton DM: Polymeric IgA and immune complex concentrations in IgA-related renal disease. Kidney international 1990, 38(2):323-31. doi:10.1038/ki.1990.204

23.Trascasa ML, Egido J, Sancho J, Hernando L: IgA glomerulonephritis (Berger's disease): evidence of high serum levels of polymeric IgA. Clinical and experimental immunology 1980, 42(2):247-54.

24.Zhang JJ, Xu LX, Liu G, Zhao MH, Wang HY: The level of serum secretory IgA of patients with IgA nephropathy is elevated and associated with pathological phenotypes. Nephrology, dialysis, transplantation: official publication of the European Dialysis and Transplant Association - European Renal Association 2008, 23(1):207-12. doi:10.1093/ndt/gfm492

25.Bene MC, Faure G, Duheille J: IgA nephropathy: characterization of the polymeric nature of mesangial deposits by in vitro binding of free secretory component. Clinical and experimental immunology 1982, 47(3):527-34. 
26.Lai KN, Tang SC, Guh JY, Chuang TD, Lam MF, Chan LY et al: Polymeric IgA1 from patients with IgA nephropathy upregulates transforming growth factor-beta synthesis and signal transduction in human mesangial cells via the renin-angiotensin system. Journal of the American Society of Nephrology: JASN 2003, 14(12):3127-37.

27.Leung JC, Tang SC, Chan LY, Tsang AW, Lan HY, Lai KN: Polymeric IgA increases the synthesis of macrophage migration inhibitory factor by human mesangial cells in IgA nephropathy. Nephrology, dialysis, transplantation: official publication of the European Dialysis and Transplant Association European Renal Association 2003, 18(1):36-45.

28.van Dixhoorn MG, Sato T, Muizert Y, van Gijlswijk-Janssen DJ, De Heer E, Daha MR: Combined glomerular deposition of polymeric rat IgA and IgG aggravates renal inflammation. Kidney international 2000, 58(1):90-9. doi:10.1046/j.1523-1755.2000.00144.x

29.van den Dobbelsteen ME, van der Woude FJ, Schroeijers WE, van den Wall Bake AW, van Es LA, Daha MR: Binding of dimeric and polymeric lgA to rat renal mesangial cells enhances the release of interleukin 6. Kidney international 1994, 46(2):512-9.

30.Matousovic K, Novak J, Yanagihara T, Tomana M, Moldoveanu Z, Kulhavy R et al: IgA-containing immune complexes in the urine of IgA nephropathy patients. Nephrology, dialysis, transplantation: official publication of the European Dialysis and Transplant Association - European Renal Association 2006, 21(9):2478-84. doi:10.1093/ndt/gfl240

31.Wang J, Xie P, Huang JM, Qu Y, Zhang F, Wei LG et al: The new Asian modified CKD-EPI equation leads to more accurate GFR estimation in Chinese patients with CKD. International urology and nephrology 2016, 48(12):2077-81. doi:10.1007/s11255-016-1386-9

32.Trimarchi H, Barratt J, Cattran DC, Cook HT, Coppo R, Haas M et al: Oxford Classification of IgA nephropathy 2016: an update from the IgA Nephropathy Classification Working Group. Kidney Int 2017, 91(5):1014-21. doi:10.1016/j.kint.2017.02.003

33.Lee SM, Rao VM, Franklin WA, Schiffer MS, Aronson AJ, Spargo BH et al: IgA nephropathy: morphologic predictors of progressive renal disease. Human pathology 1982, 13(4):314-22.

34.Novak J, Vu HL, Novak L, Julian BA, Mestecky J, Tomana M: Interactions of human mesangial cells with IgA and IgA-containing immune complexes. Kidney international 2002, 62(2):465-75. doi:10.1046/j.1523-1755.2002.00477.x

35.Novak J, Tomana M, Matousovic K, Brown R, Hall S, Novak L et al: IgA1-containing immune complexes in IgA nephropathy differentially affect proliferation of mesangial cells. Kidney international 2005, 67(2):504-13. doi:10.1111/j.1523-1755.2005.67107.x 
36.Novak J, Moldoveanu Z, Renfrow MB, Yanagihara T, Suzuki H, Raska M et al: IgA nephropathy and Henoch-Schoenlein purpura nephritis: aberrant glycosylation of IgA1, formation of IgA1-containing immune complexes, and activation of mesangial cells. Contributions to nephrology 2007, 157:134-8. doi:10.1159/0000102455

37.Valentijn RM, Radl J, Haaijman JJ, Vermeer BJ, Weening JJ, Kauffmann RH et al: Circulating and mesangial secretory component-binding $\lg A-1$ in primary IgA nephropathy. Kidney international 1984, 26(5):760-6.

38.Brandtzaeg P, Farstad IN, Johansen FE, Morton HC, Norderhaug IN, Yamanaka T: The B-cell system of human mucosae and exocrine glands. Immunological reviews 1999, 171:45-87.

39.Brandtzaeg P, Prydz H: Direct evidence for an integrated function of $\mathrm{J}$ chain and secretory component in epithelial transport of immunoglobulins. Nature 1984, 311(5981):71-3.

40.Brandtzaeg P: Transport models for secretory IgA and secretory IgM. Clinical and experimental immunology 1981, 44(2):221-32.

41.Hossain N, Khan N, Shah N, Shah T, Butt S, Khanani R: Spot urine protein-creatinine ratio and 24-h urine protein excretion: Diagnostic accuracy in women with pre-eclampsia. Pregnancy hypertension 2014, 4(1):87-90. doi:10.1016/j.preghy.2013.11.007

42.Lane C, Brown M, Dunsmuir W, Kelly J, Mangos G: Can spot urine protein/creatinine ratio replace $24 \mathrm{~h}$ urine protein in usual clinical nephrology? Nephrology 2006, 11(3):245-9. doi:10.1111/j.14401797.2006.00564.x

\section{Tables}

Table 1. Demographic and clinical characteristics of patients with IgAN and controls 


\begin{tabular}{|c|c|c|c|c|}
\hline & $\operatorname{IgAN}(n=26)$ & $\mathrm{DC}(\mathrm{n}=31)$ & $\mathrm{HC}(\mathrm{n}=32)$ & $\begin{array}{c}P \\
\text { value }\end{array}$ \\
\hline Male, n (\%) & 9 (34.6) & $16(51.6)$ & $22(68.6)$ & 0.66 \\
\hline Age (years) & $41.27 \pm 12.80$ & $45.55 \pm 16.64$ & $38.00 \pm 13.35$ & 0.29 \\
\hline BMI $\left(\mathrm{kg} / \mathrm{m}^{2}\right)$ & $24.35 \pm 3.88$ & $25.24 \pm 4.27$ & & 0.67 \\
\hline Systolic BP (mmHg) & $127.81 \pm 15.98$ & $135.94 \pm 17.08$ & & 0.10 \\
\hline Diastolic BP (mmHg) & $80.42 \pm 11.84$ & $82.74 \pm 12.66$ & & 0.52 \\
\hline MAP $(\mathrm{mmHg})$ & $96.22 \pm 12.51$ & $100.47 \pm 13.34$ & & 0.31 \\
\hline $\begin{array}{l}\text { 24-hour urine protein } \\
\text { excretion }(\mathrm{g} / 24 \mathrm{~h})\end{array}$ & $2.34 \pm 2.58$ & $5.05 \pm 4.90$ & & 0.01 \\
\hline $\begin{array}{l}\text { Serum creatinine } \\
(\mu \mathrm{mol} / \mathrm{L})\end{array}$ & $127.08 \pm 76.07$ & $108.35 \pm 73.70$ & & 0.11 \\
\hline $\operatorname{eGFR}\left(\mathrm{mL} / \mathrm{min} \cdot 1.73 \mathrm{~m}^{2}\right) \mathrm{a}$ & $70.61 \pm 34.75$ & $84.93 \pm 35.04$ & & 0.13 \\
\hline U-RBC (/uL) & $118.50 \pm 116.38$ & $1183.15 \pm 5851.43$ & & 0.11 \\
\hline Albumin (g/dL) & $38.73 \pm 5.66$ & $31.68 \pm 8.20$ & & 0.001 \\
\hline \multicolumn{5}{|l|}{$\begin{array}{l}\text { Oxford classification }{ }^{b} \text {, } \\
n \%\end{array}$} \\
\hline M0/M1 & $1(3.85) / 25(96.15)$ & & & \\
\hline E0/E1 & $22(84.62) / 4(15.38)$ & & & \\
\hline S0/S1 & $6(23.08) / 20(76.92)$ & & & \\
\hline $\mathrm{T} 0 / \mathrm{T} 1 / \mathrm{T} 2$ & $11(42.31) / 11(42.31) / 4(15.38)$ & & & \\
\hline $\mathrm{C} 0 / \mathrm{C} 1 / \mathrm{C} 2$ & $12(46.15) / 11(42.31) / 3(11.54)$ & & & \\
\hline \multicolumn{5}{|l|}{ Treatments, n (\%) } \\
\hline Corticosteroids & $4(15.4)$ & $18(58.1)$ & & 0.001 \\
\hline Immunosuppressant & $8(30.8)$ & $15(48.4)$ & & 0.12 \\
\hline ACEI/ARB & $16(61.5)$ & $17(54.8)$ & & 0.61 \\
\hline ACEI & $1(3.8)$ & $3(9.7)$ & & 0.76 \\
\hline ARB & $15(57.7)$ & $15(48.4)$ & & 0.48 \\
\hline
\end{tabular}

Data are present as mean \pm SD or median (interquartile range), or frequency in percent.

24hUPro: 24-hour urinary protein excretion; eGFR: estimated glomerular filtration rate; Urinary RBC: urinary red blood cell; SBP: systolic

blood pressure; DBP: diastolic blood pressure; MAP: mean arterial pressure; $1 \mathrm{mmHg}=0.133 \mathrm{Kpa}$; ACE-Is: angiotensin converting enzyme

inhibitors; ARBs: angiotensin II receptor blockers; M: mesangial hypercellularity score $<0.5$ (M0) or > 0.5 (M1); E: endocapillary hypercellularity absent (E0) or present (E1); S: segmental glomerulosclerosis absent (S0) or present (S1), presence or absence of podocyte hypertrophy/tip lesions in biopsy specimens with S1; T: tubular atrophy/interstitial fibrosis < $25 \%$ (T0), 26-50\% (T1), or > 50\% (T2); C: cellular/fibrocellular crescents absent (C0), present in at least 1 glomerulus (C1), in $>25 \%$ of glomeruli (C2)

a eGFR was calculated according to the Chronic Kidney Disease Epidemiology Collaboration (CKD-EPI) equation [31] 
$\mathrm{b}$ Determined in accordance with the Oxford classification [32]

\section{Figures}

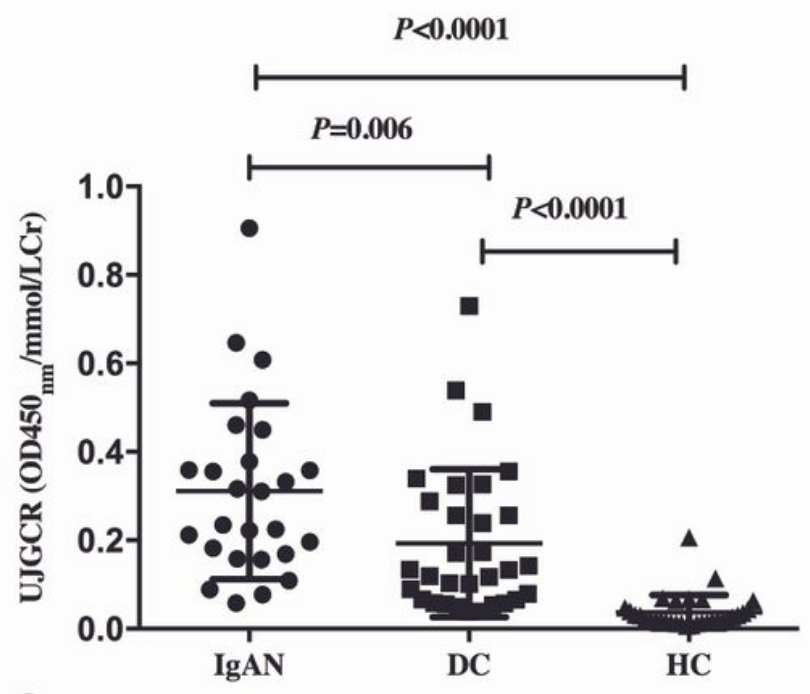

a

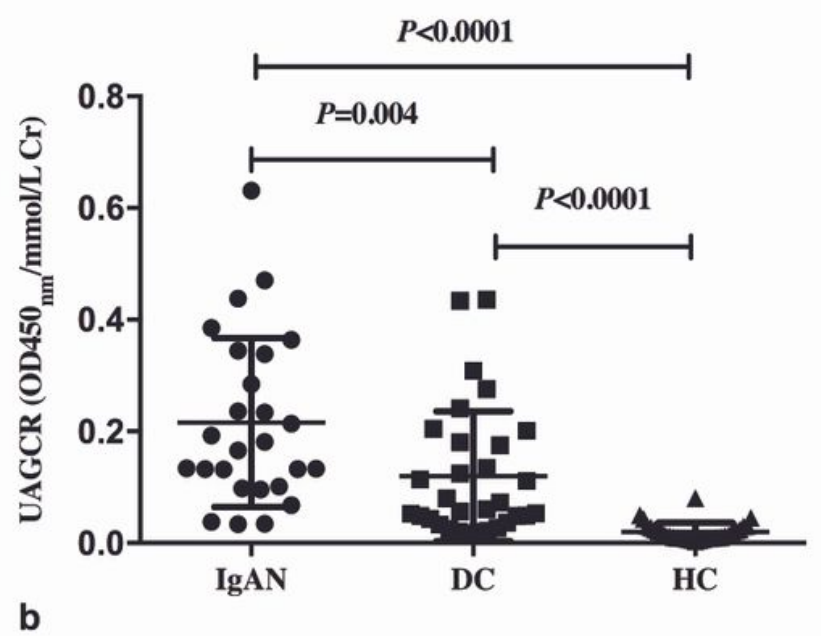

Figure 1

The levels of urinary joining chain containing IgG immune complexes/creatinine ratio and IgA-lgG immune complexes/creatinine ratio $(O D 450 \mathrm{~nm} / \mathrm{mmol} / \mathrm{L} \mathrm{Cr})$ in patients with IgA nephropathy, patients with other kidney diseases and healthy subjects. The levels of (a) UJGCR and (b) UAGCR were significantly higher in IgAN group than HC group and DC group. UJGCR: urinary joining chain containing IgG immune complexes/creatinine ratio; UAGCR: urinary IgA-lgG immune complexes/creatinine ratio; IgAN: IgA nephropathy; DC: patients with other kidney diseases; HC: healthy subjects 

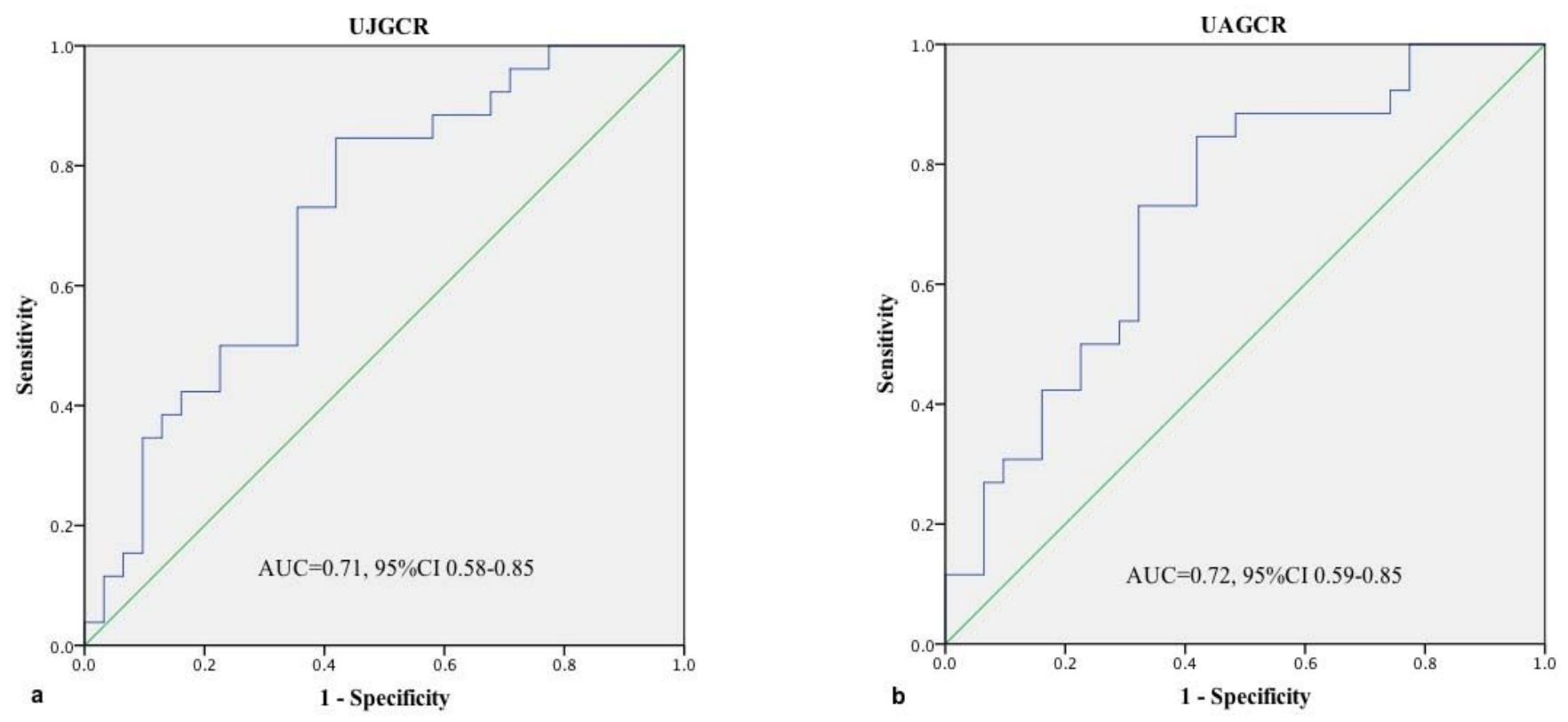

Figure 2

Receiver operating characteristic curve analysis of the prediction values of urinary joining chain containing IgG immune complexes/creatinine ratio and IgA-lgG immune complexes/creatinine ratio $(\mathrm{OD} 450 \mathrm{~nm} / \mathrm{mmol} / \mathrm{L} \mathrm{Cr})$ in patients with IgA nephropathy. ROC curve revealed good discrimination of elevated levels of (a) UJGCR and (b) UAGCR between non-IgAN patients and IgAN patients. ROC: receiver operating characteristic; AUC: area under the curve; $95 \% \mathrm{Cl}$, 95\% confidence interval; UJGCR: urinary joining chain containing IgG immune complexes/creatinine ratio; UAGCR: urinary IgA-IgG immune complexes/creatinine ratio; IgAN: IgA nephropathy Estimated glomerular filtration rate (eGFR) was calculated according to the Chronic Kidney Disease Epidemiology Collaboration (CKD-EPI) equation [31] 

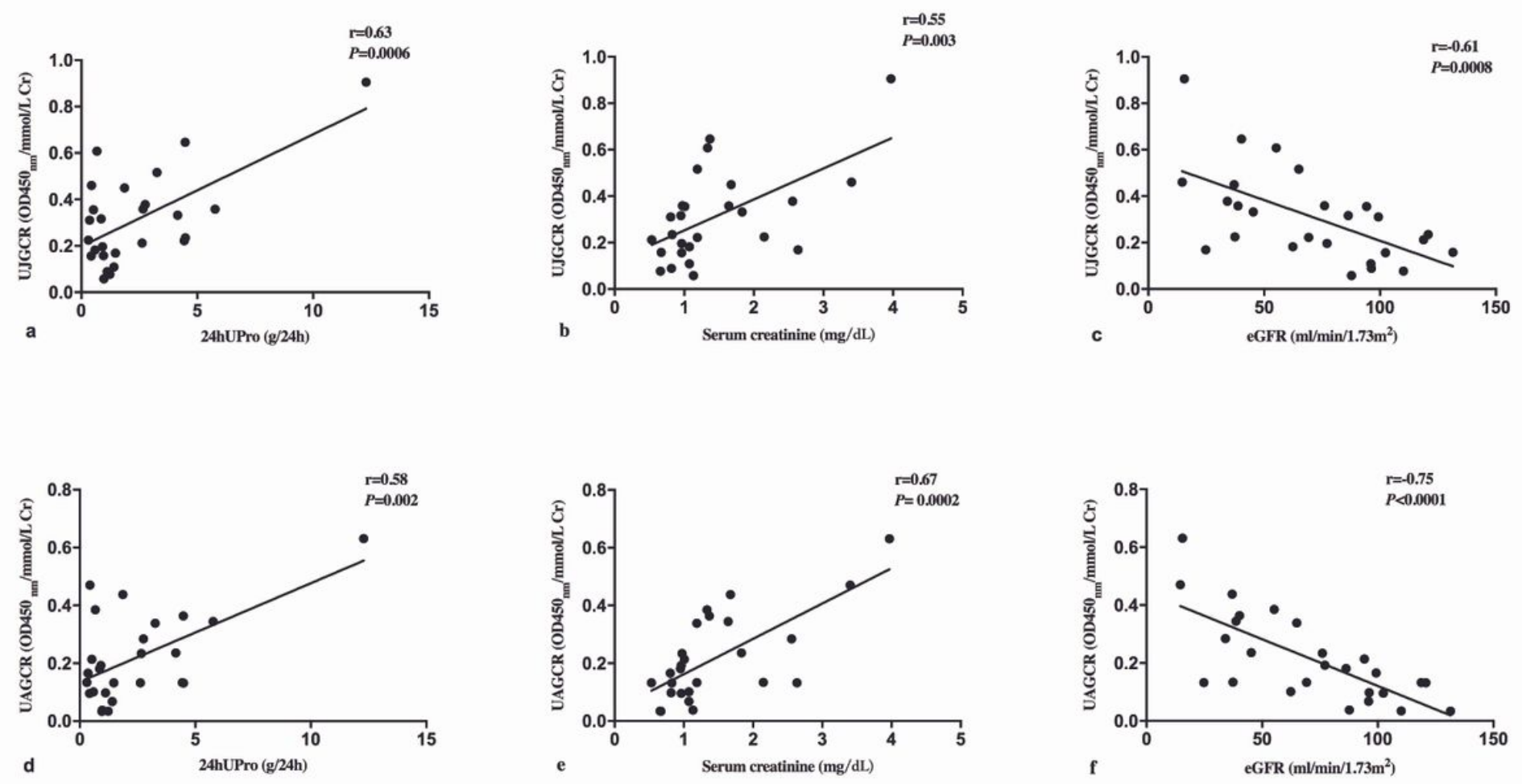

Figure 3

Correlation between the levels of urinary immune complexes and clinical parameters of patients with IgA nephropathy. ( $\mathrm{a}, \mathrm{b}$ and $\mathrm{c})$ Correlation between the levels of UJGCR and 24hUPro, serum creatinine and eGFR; ( $d$, e and f) correlation between the levels of UAGCR and 24hUPro, serum creatinine and eGFR. UJGCR: urinary joining chain containing IgG immune complexes/creatinine ratio; UAGCR: urinary IgA-lgG immune complexes/creatinine ratio; 24hUPro: 24-hour urinary protein excretion Estimated glomerular filtration rate (eGFR) was calculated according to the Chronic Kidney Disease Epidemiology Collaboration (CKD-EPI) equation [31]

\section{Supplementary Files}

This is a list of supplementary files associated with this preprint. Click to download.

- Additionalfile2.docx

- Additionalfile3.docx

- Additionalfile4.docx

- Additionalfile1.docx 